\title{
Hyperthermie - Symposium aus Anlass des 80. Jahrestages der Verleihung des Nobelpreises für Medizin an Julius Wagner-Jauregg
}

\author{
7./8. September 2007, Universitätskliniken Wien, Österreich \\ Gast-Herausgeber: B. Reiter \& R. Kleef, Wien
}

Hyperthermieverfahren im Überblick

Erich Dieter Hager, Dr. med. Dr. rer. nat. Dipl.-Phys.

BioMed Klinik, Bad Bergzabern, Deutschland

Ergebnisse von klinischen Studien zeigen, dass Hyperthermie in Kombination mit Chemo- oder Strahlentherapie eine sehr vielversprechende komplementäre Behandlungsmethode mit konsistenten und überzeugenden Resultaten ist. Die bisherigen Ergebnisse begründen die Behandlung mit Hyperthermie als ergänzende Standardbehandlung in der Krebstherapie, um das Überleben und die Lebensqualität von Krebspatienten zu verbessern. Weitere randomisierte Studien sollten dringend unterstützt werden.

\section{Julius Wagner-Jauregg (1857-1940): Porträt und kritische Würdigung}

Michael Hubenstorf, Univ.-Prof. Dr. Dr. med.

Institut für Geschichte der Medizin, Universität Wien, Österreich

Seit der Verleihung des Nobelpreises für Medizin an Julius Wagner-Jauregg im Jahr 1927 scheint seine Einschätzung durch die Nachfahren gleichsam kanonisiert. Sein Tod im Jahr 1940, Nachrufe auf beiden Seiten der damals kriegführenden Parteien und eine unkritische Kontinuität von oberflächlicher Medizinhistoriographie haben diese meist hagiographischen Einschätzungen perpetuiert. Dabei ist nicht viel mehr festzustellen als kontinuierliche Abschreibearbeit, die sich kaum je um die historischen Details gekümmert hat. Erst eine politische Neubewertung, teilweise konzentriert auf die allerletzten Lebensjahre Wagner-Jaureggs, seine Affinität zum Nationalsozialismus und sein eugenisch-rassenhygienisches Engage- ment hat in jüngster Zeit Kontroversen ausgelöst. Dies zwingt auch die Medizin und die Medizinhistoriographie, eine Neubewertung vorzunehmen. Dabei stellen sich Widersprüchlichkeiten und Ambivalenzen heraus, welche die vorherrschende Hagiographie zu sehr verdeckt hat. Die Tatsache, dass die bahnbrechende therapeutische Leistung der Malariatherapie der progressiven Paralyse bereits zweieinhalb Jahrzehnte nach ihrer Entdeckung medizinisch überholt war, ist durchaus bekannt. Weniger jedoch, dass die therapeutische Vorgehensweise von Anfang an medizinethisch umstritten war und den inhumanen Malariaexperimenten der 40er-Jahre den Weg geebnet hat. Dabei ist die Malariatherapie nur ein Komplex unter mindestens einem halben Dutzend anderer Themen, die zur vergleichenden Einschätzung der Person des Nobelpreisträgers herangezogen werden müssten. Kritische medizinhistorische Forschung hat sich bislang kaum an diese Themen herangewagt, und insgesamt ist das Lebenswerk Wagner-Jaureggs auch zu komplex, um einfache Beurteilungen nahezulegen. Dabei wäre auch davor zu warnen, ihn zu sehr vereinfachend als alleinigen Urheber heutiger Hyperthermie-Therapie darzustellen.

\section{Nano-Krebstherapie: Tumorselektive Thermotherapie mittels Nanotechnologie}

Andreas Jordan, Dr. rer. nat.

MagForce Nanotechnologies AG, Berlin, Deutschland

Bei der Nano-Krebstherapie, oder auch Thermotherapie mit magnetischen Nanopartikeln, handelt es sich um ein neues Therapiekonzept, bei dem Tumorzellen durch lokal eingebrachte Wärme geschädigt werden. Das Prinzip dieser Methode ist die direkte Einbringung einer magnetischen Flüssigkeit 
in den Tumor und ihre anschließende Erwärmung in einem magnetischen Wechselfeld. In Abhängigkeit von der Temperatur und der Einwirkdauer führt die Behandlung entweder $\mathrm{zu}$ einer direkten Schädigung der Tumorzellen oder macht diese empfindlicher gegenüber einer begleitenden Radiotherapie. Die magnetische Flüssigkeit (NanoTherm®; MagForce Nanotechnologies AG, Berlin, Deutschland) besteht aus superparamagnetischen Eisenoxid-Partikeln mit einer Eisenkonzentration von $112 \mathrm{mg} / \mathrm{ml}$. Die Nanopartikel bestehen aus einem Eisenoxid-Kern mit einem Durchmesser von zirka 15 nm und einer Umhüllung aus Aminosilanen.

Vorteile der Nanotherapie gegenüber anderen Hyperthermieverfahren sind die selektive Wärmedeposition, die freie Wahl der Zieltemperatur nach einmaliger Applikation der Nanopartikel sowie die höhere thermische Homogenität durch nahezu kontinuierliche Verteilung im Zielvolumen.

Die Ergebnisse von drei Phase-I-Studien mit insgesamt $60 \mathrm{~Pa}-$ tienten zur Machbarkeit und Tolerabilität der Behandlung des Glioblastoms, des Prostatakarzinoms sowie verschiedener anderer Tumorentitäten werden vorgestellt.

\section{Fähigkeit zu Fieber als prognostischer Faktor - Zusammenhänge mit dem Auftreten der Krebserkrankung}

Ralf Kleef, Dr. med.

Institut für Wärme- und Immuntherapie - IWIT, Wien, Österreich

Die Hyperthermie in der Onkologie erlebt international seit über 2 Jahrzehnten eine Renaissance. Zur Hyperthermie in der Onkologie (lokale Hyperthermie, Perfusionshyperthermie, Moderate- und Hochtemperatur-Ganzkörperhyperthermie [GKHT]) liegen klinische Daten vor. Bereits heute werden viele dieser Verfahren in verschiedenen Kliniken weltweit eingesetzt.

Im Mittelpunkt des Interesses stand bisher hierbei der Einfluss der Methode auf die Lebensqualität, in neuerer Zeit aber auch die mögliche Steigerung der Effektivität einer Chemound/oder Strahlentherapie sowie Kombination mit Antikörpern und Neo-Angiogenese-Inhibitoren. Daneben liegen klare immunologische Rationale vor, die wissenschaftlich untersucht und publiziert wurden und den Einsatz der moderaten Hyperthermie in der Onkologie nahelegen. Epidemiologische Untersuchungen über den Zusammenhang zwischen Krebsinzidenz und fehlendem Fieber sowie Spontanremissionen nach fieberhaften Infekten wurden vielfach beschrieben. In-vivo-Experimente mit Bakterienlysaten zeigten, dass Fieber zu einer erhöhten Expression von Hitzeschockproteinen (HSP) in Makrophagen, Blutgefäßen sowie Gefäßendothel und Enterozyten führt. Ähnliche Effekte sind auch bei einer exogenen, fieberähnlichen Hyperthermie zu beobachten.

\section{Jahre Locoregionale Tiefenhyperthermie bei Tumorpatienten: «The Graz Experience»}

Peter Kohek, Univ.-Prof. Dr. med.

AG für Hyperthermie und chirurgische Onkologie, Graz, Österreich

Es ist ein bekanntes Phänomen, dass Hitze tumorös veränderte Zellen in ihrem Wachstum hindert bzw. sie sogar abtöten kann. Die Hyperthermie wird ergänzend zu den bekannten derzeitigen Krebstherapien wie Chirurgie, Chemoimmuntherapie sowie Strahlentherapie eingesetzt, um den Therapieeffekt bei einem selektionierten Patientenkollektiv zu verstärken. Das Wort Hyperthermie stammt aus dem Griechischen und bedeutet Überwärmung (von «hyper»: zu viel, mehr und «thermos»: die Wärme).

Bei der Hyperthermie soll in bestimmten Körperarealen eine Temperatur von zirka $42{ }^{\circ} \mathrm{C}$ herbeigeführt bzw. $1 \mathrm{~h}$ lang aufrechterhalten werden. Eine signifikante Zellabtötung erfolgt ab einer Temperatur von $41{ }^{\circ} \mathrm{C}$. Die Wirkung der Hyperthermie hängt vor allem von der erreichten Temperatur ab. Ab $42,5^{\circ} \mathrm{C}$ wirkt sie direkt zytotoxisch, also zellabtötend. Bei Tumoren, welche eine schlechte Durchblutung aufweisen, können auch schon niedrigere Temperaturen zellabtötend sein, wobei sich diese Tumoren, genauso wie Tumoren mit größeren verflüssigten Nekrosezonen, sehr gut auf- bzw. überwärmen lassen.

Schon bei Temperaturen ab $40^{\circ} \mathrm{C}$ tritt ein strahlensensibilisierender Effekt ein, d.h. die zelleigene Reparatur der durch die ionisierende Strahlung der Strahlentherapie erzeugten Strahlenschäden wird in den erwärmten Zellen vermindert. Ebenso tritt bei diesen Temperaturen für bestimmte Chemotherapeutika ein sensibilisierender Effekt auf. Insbesondere bei alkylierenden Substanzen und Zystostatika, die direkt mit der DNA der Tumorzelle reagieren (z.B. Cisplatin, Carboplatin usw.). Auch weitere physiologische Effekte der Hyperthermie sind bekannt, wie die Steigerung der Durchblutung und damit das verstärkte Substratangebot im überwärmten Areal bei gut durchbluteten Tumoren.

\section{Ganzkörperhyperthermie im Deutsch-Ordens-Spital Friesach}

Klaudia Kraßnitzer, Dr. med.

Deutsch-Ordens-Spital, Friesach, Österreich

Im Rahmen der alternativmedizinischen Tätigkeiten in den Ordinationen von Dr. Klaudia Kraßnitzer und Dr. Sieghard Wilhelmer im Deutsch-Ordens-Spital-Friesach wird die Ganzkörperhyperthermie seit dem Jahr 2002 angeboten. Neben onkologischen Patienten werden hauptsächlich chronische Erkrankungen erfolgreich behandelt. Bei folgenden Krankheitsbildern konnten sowohl klinisch als auch laborchemisch nachgewiesene Behandlungserfolge erzielt werden. Die Erhebung 
technischer Daten konnte den Wirkungsmechanismus der Hyperthermie ebenso belegen.

Kurative und palliative Behandlungserfolge:

- Chronische entzündliche Darmerkrankungen: Morbus Crohn und Colitis ulcerosa.

- Erkrankungen des rheumatischen Formenkreises: Chronische Polyarthritis, systemischer Lupus erythematodes, Morbus Reiter, Polymyalgia rheumatica, CREST-Syndrom, Fibromyalgie.

- Hauterkrankungen: Psoriasis vulgaris, Neurodermitis, Akne vulgaris.

- Chronische Atemwegserkrankungen: Chronische Otomastoiditis, chronische Sinusitis, chronisch rezidivierende Bronchitis, Asthma bronchiale, chronische Tonsillitis, chronische Pharyngitis.

- Chronische Erkrankungen des Urogenitaltraktes: Chronisch rezidivierender Harnwegsinfekt, Prostatitis, Adnexitis.

- Arterielle Gefäßerkrankungen: periphere arterielle Verschlusskrankheit.

- Erkrankungen des Nervensystems: Restless-legs-Syndrom, Polyneuropathie.

- Karzinompatienten.

\section{Neuigkeiten zur Hyperthermiebehandlung bei der ankylosierenden Spondylitis (M. Bechterew)}

Uwe Lange, Prof. Dr. med.

Kerckhoff-Klinik, Bad Nauheim, Deutschland

Hintergrund: Die passive Überwärmung des Körpers (Hyperthermiebehandlung) bewirkt bei der ankylosierenden Spondylitis (AS) ohne akute Entzündungsaktivität überzufällig häufig günstige Einflüsse auf das Beschwerdebild. Die Ursachen dieses Effektes sind nach wie vor unklar.

An der antiphlogistischen Wirkung der Ganzkörperhyperthermie wie auch der analgetischen, durchblutungsfördernden und muskeldetonisierenden Wirkung besteht heutzutage kein Zweifel mehr. Wärme stellt einen somatischen Stressor dar, und Kortisol, das den Stresshormonen zugerechnet wird, zeigt einen weitgehend synchronen Anstieg unter akuten Stressreaktionen [1].

In einer Pilotstudie [2] wurde zunächst der Einfluss einer milden Ganzkörperhyperthermie (Überwärmungsbad) im Sinne einer «Stressreaktion» auf die Blutspiegel von Kortisol und Lymphozyten sowie -subpopulationen bei Patienten mit AS im Vergleich zu Gesunden untersucht. Die Arbeitshypothese konnte jedoch bei ausbleibendem Kortisolanstieg mit konsekutiver Immunsuppression im Beobachtungszeitraum (vor dem Überwärmungsbad bis zu 4,5 h danach) nicht bestätigt werden.

Aufgrund vorliegender Untersuchungen, die einen Einfluss der Wärmetherapie auf das Zytokinmilieu belegen [3], erfolg- te eine Anschlussuntersuchung zu möglichen Veränderungen des Zytokinmilieus durch serielle Hyperthermiebehandlung. Methodik: Hierzu wurden bei 24 Personen, 12 Patienten mit einer AS und 12 gesunden Probanden gleicher Altersstruktur (Durchschnittsalter 34 Jahre), seriell 9 Überwärmungsbäder im Abstand von 3 Tagen in den frühen Morgenstunden bis zum Erreichen einer Körpertemperatur von 38,5 ${ }^{\circ} \mathrm{C}$ sublingual durchgeführt (Dauer des Bades zirka 50 min). Zu Beginn des letzten Bades sowie nach 1, 6 und 24 h erfolgte die Bestimmung der Plasmazytokinspiegel von TNF- $\alpha$, Interleukin 1- $\beta$ und Interleukin 6.

Resultate: $\mathrm{Zu}$ allen 4 Messzeitpunkten zeigte sich für die bestimmten Zytokine ein hochsignifikanter Gruppenunterschied $(\mathrm{p}<0,0005)$. Bei den Patienten zeigten alle Zytokine einen signifikanten Abfall zwischen den Messzeitpunkten nach 1 und $6 \mathrm{~h}$ sowie nach 6 und $24 \mathrm{~h}$.

Diskussion: Der durch serielle, systemisch milde Hyperthermie hervorgerufene Besserungseffekt bei AS scheint aus dem Nachweis von thermischen Effekten auf das Zytokinmilieu zu resultieren, mit konsekutiven Hyperthermieauswirkungen auf Entzündungen und Immunreaktionen.

\section{Literatur}

1 Kaiser H, et al: Cortisontherapie. Corticoide in Klinik und Praxis, ed 9. Stuttgart, Thieme, 1992.

2 Lange U, et al: Einfluss der milden Hyperthermie auf die Blutspiegel von Kortisol und Lymphozytensubpopulationen bei Patienten mit ankylosierender Spondylitis und Gesunden. Phys Med Rehab Kurort 2005;L15:44-47.

3 Schmidt KL: Akutelle Aspekte der Balneotherapie rheumatischer Erkrankungen; in Karagülle MZ, Gutenbrunner C, Karagülle O (Hrsg): Balneologie und Klimatologie bei rheumatischen Erkrankungen. Positionsbestimmungen zur Jahrhundertwende. Sarow, ISMH Verlag, 2003; pp 49-58.

\section{Das fiebernde Kind - vom heilsamen Umgang mit Fieber}

Alfred Längler, Dr. med.

Gemeinschaftskrankenhaus, Herdecke, Deutschland

Fieber wird in der Regel als zu bekämpfendes Symptom im Rahmen von Erkrankungen gesehen. Kritiklose Antipyrese kann aber in bestimmten Krankheitssituationen kurz-, mittelund langfristig auch negative Effekte auf die Gesundheit bzw. Heilungschancen des Patienten haben. Einer kurzen einführenden Darstellung der Pathophysiologie des Fiebers folgt eine Schilderung positiver akuter Fiebereffekte. Ein Schwerpunkt liegt dabei auf der Darstellung des Einflusses von Fieber bei viralen Erkrankungen. So ist die Komplikationsrate bei Viruserkrankungen bei behutsamem Umgang mit Fieber möglicherweise geringer. 


\section{Hyperthermie bei entzündlichen Darmerkrankungen}

Gerfried Lexer, Prim. Dr. med.

Zell am See, Österreich

Insgesamt wurden bisher 10 Patienten mit entzündlichen Darmerkrankungen additiv mittels Hyperthermie behandelt. Es ist festzustellen, dass konventionelle Therapie mit Claversal ${ }^{\circledR}$ (Merckle Recordati GmbH, Ulm, Deutschland) und/oder Pentasa ${ }^{\circledR}$ (Ferring GmbH, Kiel, Deutschland) den Patienten immer und regelmäßig verabreicht wurde, in akuten Schüben wurde auch Cortison zusätzlich eingesetzt.

Die Patienten wurden primär einer ausführlichen endoskopischen und laborchemischen Untersuchung unterzogen.

Der Behandlungserfolg wurde auch immer wieder endoskopisch und histologisch kontrolliert.

Von den 10 Patienten hatten insgesamt 8 eine milde Colitis, 2 Patienten hatten eine schwer exazerbierte Colitis ulcerosa. Es wurden bei allen 10 Patienten Behandlungssitzungen im Abstand von 3 bzw. 2 Tagen hintereinander durchgeführt und anschließend Erhaltungssitzungen 1-mal monatlich, wobei bei gutem Verlauf in den Sommermonaten Juni, Juli, August auf Behandlungen verzichtet wurde.

Die Temperaturen wurden zwischen 38,5 und $39,5^{\circ} \mathrm{Grad}$ gewählt. Von den insgesamt 10 Patienten sind jetzt lediglich noch die 2 Patienten mit schwerer Colitis in laufenden therapeutischen Sitzungen.

Zum Nutzen und Erfolg der Hyperthermie ist zu sagen, dass sie als eine ideale Ergänzung im therapeutischen Schema der konservativen Behandlung der Colitis ulcerosa dient.

Es ist durch die Kombination aller Behandlungsmöglichkeiten zu einer deutlichen Verbesserung des Krankheitsbildes gekommen.

In Zusammenschau aller therapeutischern Möglichkeiten darf die Hyperthermie nicht als kurative Methode zur Behandlung von entzündlichen Darmerkrankungen bewertet werden.

\section{Die gesundheitsfördernde Wirkung des Fiebers in der Anthroposophischen Medizin}

René Madeleyn, Dr. med.

Filderklinik für Kinderheilkunde und Jugendmedizin, Filderstadt, Deutschland

So wie das Kind sich seelisch in der Trotzphase und der krisenhaft verlaufenden Pubertät in aggressiven Handlungen gegen seine soziale Umwelt zu behaupten lernt, ist die Möglichkeit, Fieber zu bilden, Ausdruck der Selbstbehauptungskraft des Ich im menschlichen Körper. Im Reifen des kindlichen Immunsystems lernt das Kind, sich gegen Krankheiten zu wehren. Fieber ist Symptom dieses sinnvollen Lernvorgangs.

Der praktische Umgang mit Infektionskrankheiten im Kindesalter zeigt, dass Antipyrese, sofern nicht eine schwere andere Grundkrankheit besteht, unnötig ist.
Entscheidend ist, dass den Eltern vermittelt wird, inwiefern Fieber Ausdruck einer starken Wirksamkeit kindlicher Persönlichkeitskräfte im Organismus ist.

\section{Die Malariatherapie als paradigmatische Methode der biologischen Psychiatrie}

Theodor Meißel, Prim. Dr. med. Landesklinikum Donauregion Gugging, Maria Gugging, Österreich

Schon als Assistent schlug Wagner-Jauregg 1887 in seiner Monografie «Über die Einwirkung fieberhafter Erkrankungen auf Psychosen» vor, das «Naturexperiment» der Besserung von psychotischen Zuständen im Zusammenhang mit interkurrenten fieberhaften Erkrankungen nachzuahmen, also Psychosen durch das Hervorrufen einer relativ ungefährlicheren Infektionskrankheit zu behandeln.

Er war sich der Problematik eines solchen therapeutischen Eingriffes wohl bewusst, arbeitete dementsprechend 30 Jahre lang an der Überprüfung seiner Hypothesen, bis er im Juni 1917 das Blut eines malariakranken Soldaten von der mazedonischen Front vier paralytischen Patienten impfen ließ.

Dieses therapeutische Aufgreifen eines «Fingerzeiges der Natur» erwies sich als erfolgreich, wenngleich es einige Todesfälle, vor allem auf Grund von Schlampereien von Mitarbeitern, gab. Meist kam es zu überzeugenden Besserungen des Krankheitsverlaufes.

Wagner-Jauregg war als Wissenschaftler primär therapeutisch interessiert, setzte immer wieder an einem konkreten, aufmerksam und kreativ erfassten klinischen Anlass an, konnte auf der Basis seiner gründlichen naturwissenschaftlichen Schulung eine Einzelbeobachtung als «Fingerzeig der Natur», als exemplarischen Fall erfassen - eine Herangehensweise, die durchaus der Sigmund Freuds gleicht.

Ideologisch und durch persönliche Beziehung war WagnerJauregg dem Nationalsozialismus zumindest sehr nahestehend, bei affektivem Bedarf war er auch offen antisemitisch, wenngleich er sich in seinen schriftlichen Arbeiten nie rassistisch äußerte. Wagner-Jauregg war auch kein Vertreter der Degenerationstheorie in der Psychiatrie, relativierte genetische Aspekte unter Hinweis auf soziale Momente, engagierte sich zwar für Eugenik, relativierte allerdings die Bewertungen hereditärer Faktoren, indem er sich vor allem auch gegen eine Einteilung der Menschen in belastete und unbelastete wandte, denn wir alle seien mehr oder wenig belastet, so dass man ihn auch nicht als Wegbereiter der späteren Nazipsychiatrie und deren Verbrechen ansehen kann.

Die Malariatherapie als paradigmatische Methode biologischer Psychiatrie verweist so bei allem Fortschritt, den sie bedeutete und den sie in die Wege leiten konnte, auch auf die Grenzen einer sich im Somatischen begrenzenden, reduzierten Naturwissenschaftlichkeit. 


\section{Vom kalten und vom heißen Fieber - die Bedeutung der Körperwärme in Medizingeschichte und Ethnomedizin}

Armin Prinz, Univ. Prof. Dr. phil. Dr. med.

Institut für Geschichte der Medizin der Universität Wien,

Österrreich

Als die Körpertemperatur noch nicht gemessen, sondern nur gefühlt werden konnte, war «Fieber» der Ausdruck für alle krankhaften Zustände, bei denen ein beschleunigter Herzschlag Leitsymptom ist. Fühlte sich die Haut des Kranken kalt an, wurde es «kaltes Fieber» genannt, wenn sie warm war, «heißes Fieber». So wurden vor allem emotionale Belastungen, die Tachykardie und Zittern auslösten, als «kaltes Fieber» bezeichnet. Dieser Zusammenhang wird etwa in dem ungarischen Märchen «Der Tod und die Alte», in dem ein Kampf mit dem Tod beschrieben wird, mit dem Satz deutlich: «Ihr ist der Schrecken so in die Glieder gefahren, dass sie bald das kalte Fieber befallen hätte.» In diese Vorstellung vom «kalten Fieber» fielen jedoch nicht nur die Folgen von Emotionen, sondern alle fieberhaften Erkrankungen, die von Schüttelfrost begleitet waren. Auch die Malaria wurde dazu gezählt, zeichnet sie sich doch beim Fieberanstieg durch starken Schüttelfrost bei kalter Haut aus. Die offizielle Medizin bezeichnete bald ausschließlich die Malaria als «kaltes Fieber», ein Ausdruck, der bis zum Beginn des vorigen Jahrhunderts auch in Lehrbüchern üblich war. Mit dem Aufkommen der Chinarinde als Fiebermittel, wurden zuerst alle Tachykardien mit dieser südamerikanischen Pflanzendroge behandelt. Dies besonders, wenn das «kalte Fieber» mit starkem Schüttelfrost, eben der Initialphase der Malaria, verbunden war. Es hat sich dadurch praktisch automatisch die Indikation Wechselfieber für die Chinarinde ergeben, auch wenn in der volkstümlichen Vorstellung noch keine Differenzierung zwischen der Symptomatik der Malaria und jener der seelischen Belastung gemacht wurde, wie bei Friedrich Schiller in seinem Brief an Reimann am 5. Mai 1784 nachzulesen ist: «Den ganzen Winter hindurch verließ mich das kalte Fieber nicht ganz. Durch Diät und China zwang ich zwar jeden neuen Anfall, aber die schlimme hiesige Luft, worin ich noch Neuling war, und meine von Gram gedrückte Seele machten ihn bald wiederkommen.» Eine Überdosierung mit Chinin kann aber auch iatrogen eine Tachykardie auslösen, ein Umstand, der möglicherweise beim Selbstversuch Hahnemanns eingetreten ist und ihn zu dem Schluss gelangen ließ, die Chinarinde könnte in homöopathischen Mengen Fieber bekämpfen.

\section{Lokoregionale Elektrohyperthermie bei Gliomen}

Hüseyin Sahinbas, Dr. med.; Dietrich H.W. Grönemeyer, Prof. Dr. med.

Grönemeyer-Institut für MikroTherapie,

Universität Witten/Herdecke, Bochum, Deutschland

Vermutlich ist die Abwandlung der Blut-Hirn-Schranke durch elektromagnetische Felder im Zusammenspiel mit der direkten, vom elektromagnetischen Feld verursachten Wärme [1] der wichtigste Faktor für den Erfolg der Elektrohyperthermie (EHY; regionale Radiowellen-Tiefenhyperthermie).

Zielstellung: Das primäre Ziel dieser Studie bestand in der Darstellung der Therapieverträglichkeit der Elektrohyperthermie für Patienten mit fortgeschrittenen Hirntumoren und der Verlängerung der medianen Gesamtüberlebenszeit.

Patienten und Methoden: Unsere retrospektive Studie erfolgte zwischen Februar 2000 und April 2007 für Patienten mit inoperablen, teilresezierten oder rezidivierenden Gliomen (WHO-Grad III und IV) in Progress nach Radiatio und/oder Chemotherapie.

In die Auswertung waren 220 Patienten einschließlich Kinder eingeschlossen: 30\% anaplastisches Astrozytom (WHO III), $60 \%$ Glioblastoma multiforme (WHO IV), zirka 10\% Metastasen, Median Alter: 39,7 Jahre. Alle Patienten waren umfangreich vorbehandelt worden. Die Elektrohyperthermie wurde über 4-12 Wochen 3-mal pro Woche jeweils für $1 \mathrm{~h}$ als Mono- oder kombinierte Therapie (Chemotherapie, Strahlentherapie sowie Supportivmedikationen, z.B. Weihrauch, Vitamine, Selen) angewendet [2-6].

Ergebnisse: Die historische Referenz der Gesamtüberlebenszeit von der Diagnosestellung an beträgt für Astrozytome und Gliome vom WHO-Grad III und IV in unserem Institut 11,42 Monate. Dies stimmt gut mit der relevanten Literatur überein $[2,3,7-9]$.

Die Gesamtüberlebenszeit $[1,10]$ in unserem Institut mit Elektrohyperthermie steigt um 38\% beim diffusen Astrozytomen, um 146\% beim anaplastischem Astrozytom und um 57\% beim Glioblastom. Besonders Bemerkenswert: 13 von $92 \mathrm{~Pa}-$ tienten mit Glioblastom leben noch nach 3 Jahren. Normalerweise erlebt dies fast niemand.

Zusammenfassung und Schlussfolgerungen: Elektrohyperthermie ist in Kombination oder in Ausnahmefällen als Monotherapie eine geeignete zusätzliche Behandlungsmöglichkeit bei fortgeschrittenen inoperablen Hirntumoren oder Metastasen [11].

In einigen Fällen konnten eine komplette/partielle Remission und/oder eine signifikante Verzögerung des Tumorwachstums gezeigt werden. Diese Kombinationstherapie zeigt eine Verbesserung der Lebensqualität und Verlängerung der medianen Gesamtüberlebenszeit [12]. Die angewendete Hyperthermiebehandlung wurde von den Patienten in fortgeschrittenen Tumorstadien und in pädiatrischen Fällen gut toleriert. 


\section{Literatur}

1 Dani A, et al: Advanced local cancer treated with local hyperthermia. Presented in Hyperthermia Seminar. October 26-27, 2003, Cologne, Germany.

2 Hager ED, et al: The treatment of patients with high-grade glioma with deep RFHyperthermia. Presented in ASCO 2003, Chicago, USA, p 470

3 Hager ED, et al: Response and survival of patients with gliomas grade III/IV treated with RF capacitive-coupled hyperthermia. ICHO Congress, St. Louis USA 2004

4 Hager ED, et al: Clinical response and overall survival of patients with recurrent gliomas Grade III/IV treated with RF Deep Hyperthermia - an update. ICHS Conference, Shenzhen, China, 2004.

5 Ebert PS, Salcman M: Differentiation therapy is potentiated by chemotherapy and hyperthermia in human and canine brain tumor cells in vitro. Neurosurgery 1994;34(4):657-64.

6 Pagani E, Falcinelli R, Repponi R, et al: Combined effects of temozolamidehyperthermia on cell growth and O6-Alkylguanine-DNA alkyltransferase (OGAT) activity of human melanoma cell lines. Anticancer Res 1998;18(237): 4807-5006.

7 De Vita H, et al: 5-Jahres Heilungsrate bei Krebserkrankungen in Europa, ed 6. 2002, p 2120, tables 2-15, 43.

8 Walker MD, Alexander E Jr, Hunt WE, et al: Evaluation of BCNU and/or radiotherapy in the treatment of anaplastic gliomas: A cooperative clinical trial. J Neurosurg 1978;49:333-343.

9 Ries LAG, Eisner MP, Kosary CL, et al (eds): SEER Cancer Statistics, 1973 1998. Bethesda, MD, National Cancer Institute, 2001.

10 Szasz A, Sahinbas H, Dani A: Electro-hyperthermia for anaplastic astrocytoma and glioblastoma multiforme. ICACT, Paris, 9-12 February, 2004

11 Fiorentini G, et al: A phase II clinical study on relapsed malignant gliomas treated with electro-hyperthermia. In Vivo 2006;20(6A):721-724.

12 Friedlander DR, Zagzag D, Shiff B, et al: Migration of brain tumor cells on extracellular matrix proteins in vitro correlates with tumor type and grade and involves alphaV and beta1 integrins. Cancer Res 1996;56(8):1939-47.

\section{Explorative Untersuchungen zum Infrarot- Niedertemperatur-Prinzip: Thermoregulation und thermische Belastung der Haut}

Ralf Kleef, Dr. med.; Andreas Zimmermann, Dr. rer. nat.; Otto Pecher, Dr. med.

Institut für Wärme- und Immuntherapie - IWIT, Wien, Österreich

Die Effekte von Infrarotkabinen sind als Reaktion auf die Wärmeapplikation erklärt. Die Strahlungsart (A, B oder C) ist dabei von untergeordneter Bedeutung. Grob lassen sich zwei Konzepte - Saunaprinzip oder das der Niedertemperatur-Infrarottechnik (NIT) - unterscheiden $[1,2]$.

Hautbelastung und Wärmeregulation wurden während einer 40-minütigen NIT-Anwendung in einer Physiotherm IRKabine (Pro-Fit 2 deluxe) untersucht. 7 gesunde männliche Probanden nahmen teil.

Die Kabinenlufttemperatur wurde auf $36{ }^{\circ} \mathrm{C}$ konstant gehalten, die $\mathrm{CO}_{2}$-Konzentration auf maximal 1000 ppm begrenzt. Der Strahler wurde 12 min mit $100 \%$ und anschließend mit 83\% Leistung gefahren (Standard). Die Temperatur des Strahlers (bei $100 \%$ ca. $100 \mathrm{~mW} / \mathrm{cm}^{2}$ in $10 \mathrm{~cm}$ Abstand) wurde dokumentiert.

Zur Erythemberechnung wurde die Haut vor und bis $2 \mathrm{~h}$ nach der Anwendung optisch-spektroskopisch vermessen [3, 4]. Neben einer berührungslosen Messung der Hauttemperatur im Rückenbereich [5] zeichneten Kontaktsensoren die Tem- peraturen im Rücken- (4 Messstellen) und Brustbereich sowie an beiden Ober- und Unterschenkeln auf. Ferner wurden Blutdruck, Rektaltemperatur, pO2, Puls und EKG dokumentiert.

Die max. Hauttemperatur wurde im Rückenbereich nach 5 min erreicht und lag im Mittel $4{ }^{\circ} \mathrm{C}$ unter dem kritischen 30-Minuten-Wert von $46,6{ }^{\circ} \mathrm{C}$ [6]. Die Hautrötung war nach $2 \mathrm{~h}$ abgeblasst [6]. Die Körperkerntemperatur stieg im Mittel um $0,2-0,3{ }^{\circ} \mathrm{C}$ an [7]. RR und Puls nahmen leicht ab. Die $\mathrm{O}_{2-}$ Sättigung sank um 1\%. Die Hauttemperatur an den Extremitäten ereichte nach ca. 10 min ein Maximum.

Die Sicherheitsgrenzwerte wurden weit unterschritten. Das NIT regt eine TR entsprechend erhöhte, körpereigene Wärmeproduktion an. Eine schonende Adaption des Organismus ist möglich. Die Erhöhung von Durchblutung und Versorgung ist dadurch möglicherweise verträglicher und effektiver. Detailliertere Untersuchungen sind erforderlich.

\section{Literatur}

Sohar E, et al: Effects of exposure to Finnish sauna. Isr J Med Sci 1976;12:12751282.

2 Leppaluoto J: Human thermoregulation in sauna. Ann Clin Res 1988;20:240-243. Sprigle S, et al: Analysis of localized erythema using clinical indicators and spectroscopy. Ostomy Wound Manage 2003;49:42-52.

4 Fullerton A, et al: Guidelines for measurement of skin colour and erythema. A report from the Standardization Group of the European Society of Contact Dermatitis. Contact Dermatitis 1996;35:1-10.

5 Krause BF: Accuracy and response time comparisons of four skin temperaturemonitoring devices. Nurse Anesth 1993;4:55-61.

6 Wienert V, et al: [Local thermal stress tolerance of human skin]. Anasth Intensivther Notfallmed 1983;18:88-90.

7 Explorative Untersuchungen zur Infrarot-Niedertemperaturtechnik mit ILCS (Instant leukocyte culture system) zu physiologischen und immunmodulierenden Effekten. Pressekonferenz Dez. 2005, Wien. 